\title{
Validation of an In Vitro Model to Study Human Cartilage Responses to Compression
}

\author{
Natalia de Isla ${ }^{1}$, Céline Huselstein ${ }^{1}$, Didier Mainard ${ }^{1,3}$, Jean-François Stoltz ${ }^{1,2}$ \\ ${ }^{1}$ CNRS UMR 7561, Biopôle, Faculté de Médecine, Université de Lorraine, Vandoeuvre, France \\ ${ }^{2}$ UTCT, CHU de Nancy, Vandoeuvre, France \\ ${ }^{3} \mathrm{COT}, \mathrm{CHU}$ de Nancy, Vandoeuvre, France
}

Received 2012

\begin{abstract}
The aim of this work was to develop an in vitro model to study mechanical compression effects on cartilage. A pressure-controlled compression device was used in this study. Cartilage explants obtained from human knee were compressed at $1 \mathrm{MPa} / 1 \mathrm{~Hz}$ for $7 \mathrm{hours}$ (30 min ON, 30 min OFF) under normoxia $\left(5 \% \mathrm{CO}_{2}, 21 \% \mathrm{O}_{2}\right)$ or hypoxia $\left(5 \% \mathrm{CO}_{2}, 5 \% \mathrm{O}_{2}\right)$. Cell viability was analyzed while nitric oxide (NO) and glycosaminoglycans (GAG) release was assayed in culture media. Mechanical stimulation increased NO production and GAG release by human cartilage explants under normoxia and hypoxia culture. In normoxia and hypoxia conditions, mechanical stimulation alters human OA cartilage metabolism. There is also, an increase in matrix degradation after compression, as shown by levels of GAG found in culture media. This study put in evidence the importance of mechanical compression in the progression of the osteoarthritis and present and in vitro model for mechanobiological and pharmacological studies.
\end{abstract}

Keywords: Cartilage; Compression; In Vitro Model; Nitric Oxide; GAG

\section{Introduction}

Cartilage is an avascular tissue submitted in vivo to mechanical stimuli. These mechanical stimuli result from a complex combination of tension, shearing and compression forces, the latter being the most important within the cartilage [1]. The compressive forces exerted on the surface of the articular cartilages are variable according to the weight of the individual, his muscular tension and its physical-activity. Thus for example the average pressure being exerted on the hip is of $0.7 \mathrm{MPa}$ but during physical exercises, it can reach 5 to $10 \mathrm{MPa}$. These mechanical forces affect the extracellular matrix as well as the chondrocyte metabolism [2-9]. During immobilization, the capacities of synthesis of chondrocytes as well as the thickness of the cartilage decrease. In the same way, in the zones subjected to maximal forces, the balance between the anabolism and the catabolism of the cartilage are disturbed.

It is know that physiological loading of articular cartilage is necessary to maintain normal joint function. Articular chondrocytes transform mechanical signals into biochemical ones to maintain the integrity of their extracellular matrix [10-12]. Several studies investigated the effect of mechanical stimulation on chondrocyte metabolism. In general, static compression decreases biosynthetic activity compared to unloaded tissue while dynamic compression has been found to stimulate, inhibit or have no effect on biosynthetic activity depending on the loading frequency and amplitude [13-15]. Other studies have shown that cyclic tensile strains of low magnitude (3-8\% equibiaxial strain) and physiological levels of cyclic compressive forces (15\% compression) elicit an anabolic response [16, 17], while strains of high magnitude (10-15\% equibiaxial strain) initiate cartilage damage.

Most of the studies investigated the effects of mechanical compression on osteoarthritis (OA) development and use healthy cartilage principally from animal origin. In this work we aimed to investigate the response of human cartilage from osteoarthritic patients to dynamic unconfined compression. The aim of this work was to develop an in vitro model to study mechanical compression effects on cartilage and to define experimental protocols to be used in cartilage mechanobiology. This model could be used in pathophysiological or pharmacological studies of cartilage.

\section{Materials and Methods}

\subsection{Cartilage Explants}

Articular cartilage was obtained from preserved areas of femoral condyles of patients undergoing arthroplasty for $\mathrm{OA}$ at the Department of Orthopaedic Surgery, CHU Nancy, France. Samples from 10 patients (6 women and 4 men, $64 \pm 7$ years) were used. Cartilage was separated from the subchondral bone using a scalpel. Cylindrical explants ( $5 \mathrm{~mm}$ in diameter) were harvested using a sterile biopsy punch (Stiffel, France) and immediately incubated in culture medium (DMEM-F12) supplemented with $10 \%$ heat inactivated fetal bovine serum, $1 \%$ of antibiotics/antimycotic solution, and $2 \mathrm{mM}$ Glutamine at $37^{\circ} \mathrm{C}$, $5 \% \mathrm{CO}_{2}$. Test and control explants were paired at harvest and originated from adjacent sites on the joint surface. All compression experiments were performed after allowing explants to equilibrate in culture for 72 hours after harvest.

\subsection{Mechanical Stimulation}

A FX-4000CTM Flexcercell ${ }^{\circledR}$ Compression Plus TM System (Flexcell International, Hillsborough, NC) was used to apply dynamic compression. Explants were placed in one well of a 
Biopress culture plates (Flexcell International) and mounted within the apparatus. The plates consist of a 6 well plates containing a flexible silicone rubber membrane at the bottom. The explant is putted on the plastic disc, into the Foam Sample Holder and the piston of the Stationary Platen is moved until it become in contact with the explants. A calibrated air pressure was applied to the membrane to obtain a compressive stress $(\sigma)$; determined from the applied force $(\mathrm{F})$ and the initial crosssectional area (A) of the explant using the equation $\sigma=$ F/A (Figure 1). Two millilitres of culture medium were introduced into each well. Explants were subject to unconfined compression at compressive stress amplitude of $1 \mathrm{MPa}$ at $1 \mathrm{~Hz}$ for 7 hours in an intermittent manner (30 min ON, $30 \mathrm{~min}$ OFF) in a humified incubator at $37^{\circ} \mathrm{C}, 5 \% \mathrm{CO}_{2}$. Unloaded (control) explants were incubated under the same conditions. Tests were performed in order to calculate the pressure applied to the sample by using a force sensor (XFL 205 R, FGP Sensors \& Instrumentation) instead the sample and to be sure that all culture wells on each of the 4 compression plates of the device were subject to the same strain (Figure 1).

To analyze the effect of oxygen tension in NO production and GAG release, experiments were performed in a humidified incubator at $37^{\circ} \mathrm{C}, 5 \% \mathrm{CO}_{2}, 5 \% \mathrm{O}_{2}$. Explants were left overnight in hypoxia before each experiment in order to let cells to adapt their metabolism.

\subsection{Viability Assay}

Cell viability was determined in cartilage explants using the fluorescent probes Propidium Iodure (PI) and SYTO16 (both from Molecular Probes). The membrane-permeable SYTO16 labels live and dead cells to yield cytoplasmic and nuclei green fluorescence, whereas the membrane-impermeable propidium iodide labels nucleic acids of membrane-compromised cells with red fluorescence. After each experiment, the loaded and

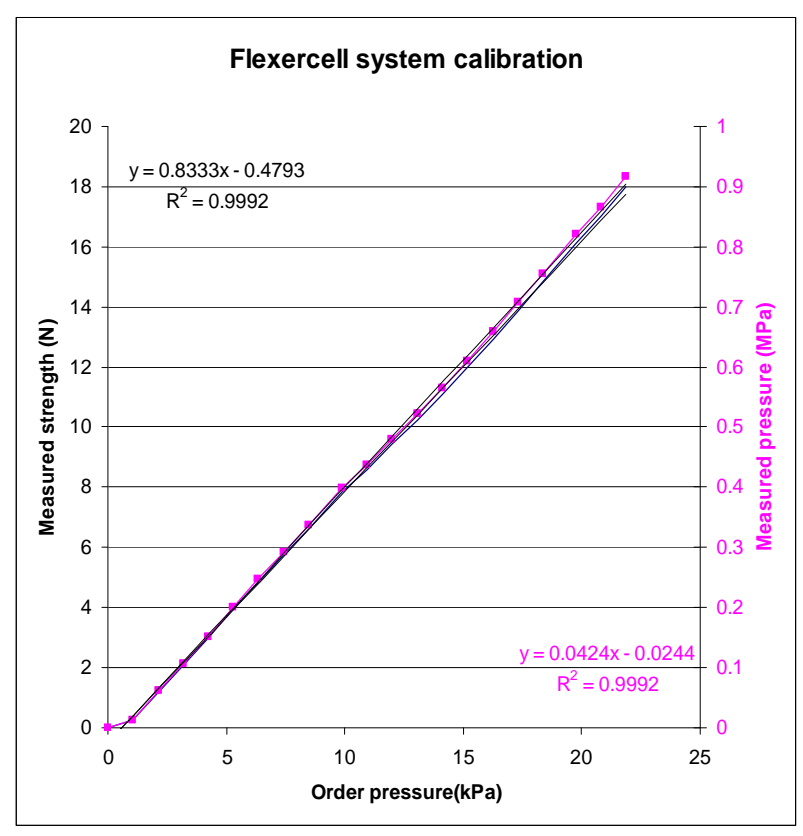

Figure 1. Calibration of the compression device using a force sensor. unloaded (control) explants were washed in DMEM W/O phenol red for $5 \mathrm{~min}$ and then sectioned perpendicular to the articular surface into 1-mm thick slices using parallel razor blades. The tissue sections were incubated in the SYTO16+PI solution for $5 \mathrm{~min}$ in a dark environment and then washed twice $(5 \mathrm{~min}$ each) in DMEM W/O phenol red to remove free dye from the tissue matrix. The chondrocytes within the cartilage matrix were then viewed using a fluorescence confocal microscope (LEICA) in sequential mode (excitation/emission: $488 \mathrm{~nm} /$ $520 \mathrm{~nm}(1) ; 545 / 633$ (2)) to simultaneously observe green and red fluorescence. Green cells are viable and yellow cells are dead. The percentage of dead cells was calculated by counting the total number of cells and the number of yellow cells in five random, non-contiguous fields.

\subsection{Measurement of GAG Release}

GAG levels in the culture medium were determined by the amount of polyanionic material reacting with DMMB (Polysciences, USA). Explants supernatants were removed and 125 $\mu 1$ were combined with $200 \mu \mathrm{l}$ of DMMB solution. Samples were examined spectrophotometrically at $525 \mathrm{~nm}$. For this assay, standards prepared with control media and chondroitin sulphate C (Sigma, France) were used. Results are reported as $\mu \mathrm{g}$ GAG per mg of wet weight of tissue.

\subsection{Determination of NO}

NO production was measured by estimating the stable NO metabolite, nitrite, in conditioned medium using a spectrophotometric method based on the Griess reaction (Griess Reagent Kit for Nitrite Determination, Molecular Probes). Following culture of the cartilage explants for the times indicated, $150 \mu \mathrm{l}$ of the culture supernatants or sodium nitrite standard dilutions were mixed with $20 \mu \mathrm{l}$ Griess reagent ( $1 \%$ sulfanilamide, $0.1 \%$ naphthyl ethylenediamine dihydrochloride, and 5\% H3PO4) and incubated for $30 \mathrm{~min}$ at room temperature. Nitrite concentrations were determined by measuring absorbance at $570 \mathrm{~nm}$ in a Microplate reader (BioRad, U.S.A.). Values are expressed as $\mu \mathrm{M}$ nitrite released per mg of wet weight of tissue.

\section{Results}

Cell viability in response to compression. Fluorescence staining indicated that cell death was confined to the cut edge and to the superficial zone in uncompressed control samples. In mechanically loaded explants, cell death was evident also in the intermediate region of the explants. When compared the superficial zone in unloaded and compressed samples, the percentage of dead cells is higher $(p<0.05)$ in compressed explants $(59 \%)$ than in unloaded ones $(23 \%)$. Similarly, the percentage of dead cells in the deep zone is higher $(\mathrm{p}<0.05)$ in compressed samples $(39 \%)$ than in unloaded samples $(20 \%)$.

NO production and GAG release after compression. The mechanical compression used in this in vitro study affects the NO production and GAG release from human cartilage explants. As shown in Figure 2, NO increases in culture medium of compressed explants after 7 hours of intermittent compression when compared to uncompressed explants. In parallel, conditioned medium from compressed and uncompressed cartilage 
explants was analyzed for sulphated GAG content after 7 hours. The results presented in Figure 3 showed that mechanical stimulation increase GAG release in culture medium of compressed explants when compared to uncompressed ones. We next investigated the effect of oxygen tension in the level of NO production and GAG release in response to mechanical compression. Under static conditions, NO production increased under hypoxia when compared to normoxia conditions (increase of $202 \pm 51 \%, p<0,05)$. Moreover, mechanical compression significantly increased NO production (Figure 2) under hypoxia. Furthermore, under static conditions GAG release under hypoxia did not change when compared to normoxia conditions. Mechanical compression significantly increased GAG release (Figure 3) under hypoxia although the increase is less important than under normoxia.

\section{Discussion}

In this study, the effect of unconfined compression on cartilage explants from human osteoarthritic knee was studies. Results showed that in this in vitro model, mechanical compression increased NO production and GAG release under normoxia and hypoxia conditions.

Under physiological conditions, large forces which are the result of normal joint movements are applied to articular cartilage. Mechanical load has been demonstrated in many in vivo and in vitro investigations to be an important factor affecting the health of articular cartilage and consequently the function of

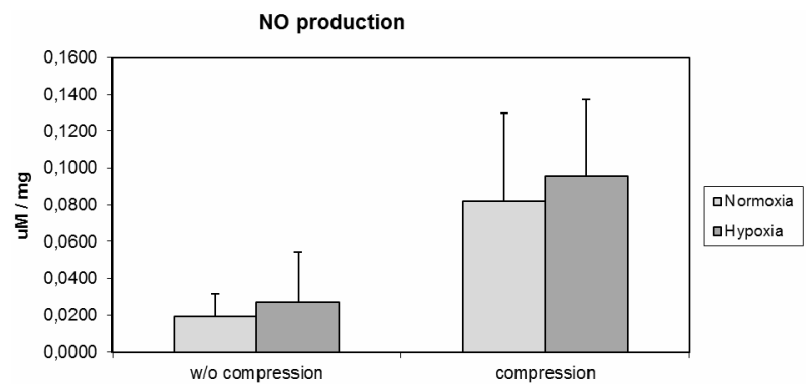

Figure 2. Production of Nitrite $(\mu \mathrm{M} / \mathrm{mg}$ wet weight) by articular cartilage explants compressed at $1 \mathrm{MPa}, 1 \mathrm{~Hz}$ for $7 \mathrm{~h}$ (30 min on, 30 min off) under normoxia conditions $\left(21 \% \mathrm{O}_{2}\right)$ or under hypoxia conditions $\left(5 \% \mathrm{O}_{2}\right)$. Data are presented as mean \pm S.D. of 3 independent experiments with $n=3 /$ group/experiment, $p<0.05:$ w/o compression vs compression.

GAG Release

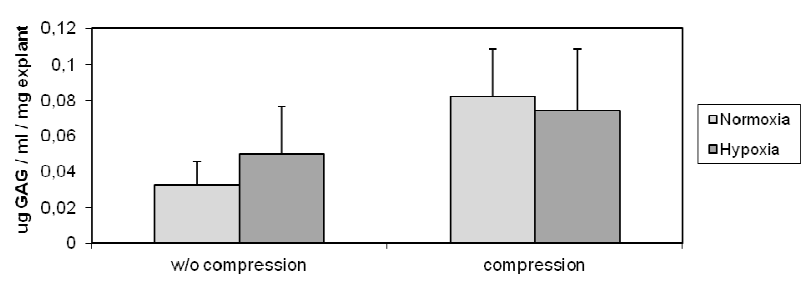

Figure 3. GAG released ( $\mu \mathrm{g} / \mathrm{mg}$ wet weight) in culture medium of articular cartilage explants compressed at $1 \mathrm{MPa}, 1 \mathrm{~Hz}$ for $7 \mathrm{~h}$ (30 min on, $30 \mathrm{~min}$ off) under normoxia $\left(21 \% \mathrm{O}_{2}\right)$ or hypoxia $\left(5 \% \mathrm{O}_{2}\right)$. Data are presented as mean \pm S.D. of 3 independent experiments with $\mathrm{n}=3$ /group/experiment, $p<0.05$ (w/o compression vs compression). the diarthrodial joint and the progression of joint degeneration $[18,19]$. The effect of the stimulation is strongly dependent on magnitude and frequency of the applied load. In the in vitro model used in this study, unconfined compression was used. Under this condition, the construct is free to expand laterally and is subject to both compressive strains (along the axial direction) and tensile strains (along the radial and circumferential directions). This loading regime represents a physiologic loading environment and produces a more uniform mechanical signal throughout the thickness of a cylindrical cartilage sample than that of confined compression (where radial expansion is prevented).

In cartilage, chondrocytes exhibit a predominantly anaerobic metabolism because they are in hypoxia under physiological conditions. The hypoxic conditions are further enhanced during $\mathrm{OA}$ because the oxygen consumption of the synovium from $\mathrm{OA}$ patients is elevated and the synovial fluid $\mathrm{O}_{2}$ tension is decreased compared with that of normal synovial fluid. In conclusion, the standard cell culture conditions with $21 \%$ oxygen tension do not mimic the physiological situation within cartilage. In this study, mechanical compression increased NO production and GAG release when experiences were performed at $5 \% \mathrm{O}_{2}$ but we noted that the increase is lower than at $21 \% \mathrm{O}_{2}$.

In conclusion, this study shows that high levels of compression increase NO production and GAG release by human OA under normoxia and hypoxia. In an in vitro model we observed that OA cartilage is sensitive to mechanical stimulation and show the importance of mechanical stimulation in the progression of the osteoarthritis.

\section{REFERENCES}

[1] Wong M and Carter DR. Articular cartilage functional histomorphology and mechanobiology: a research perspective. Bone 2003; 33:1-13.

[2] Buschmann MD, Gluzband YA, Grodzinsky AJ, Hunziker EB. Mechanical compression modulates matrix biosynthesis in chondrocyte/agarose culture Journal of Cell Science 1995; 108: 1497-1508.

[3] Chowdhury TT, Bader DL, Shelton JC, Lee DA. Temporal regulation of chondrocyte metabolism in agarose constructs subjected to dynamic compression. Arch Biochem Biophys 2003; 417: 105-11.

[4] Plumb MS and Aspden RM. The response of elderly human articular cartilage to mechanical stimuli in vitro. Osteoarthritis and Cartilage 2005;13:1084-1091.

[5] Madhavan S, Anghelina M, Rath-Deschner B, Wypasek E, John A, Deschner J, Piesco N, Agarwal S. Biomechanical signals exert sustained attenuation of proinflammatory gene induction in articular chondrocytes. OsteoArthritis and Cartilage 2006; 14: 1023-1032.

[6] Lee DA, Bader DL. Compressive strains at physiological frequencies influence the metabolism of chondrocytes seeded in agarose. J Orthop Res 1997;15:181-188.

[7] Gassner R, Buckley MJ, Georgescu H, Studer R, Stefanovich-Racic M, Piesco NP, Evans CH, Agarwal S. Cyclic Tensile Stress Exerts Antiinflammatory Actions on Chondrocytes by Inhibiting Inducible Nitric Oxide Synthase. The Journal of Immunology 1999; 163: 2187-2192.

[8] Démarteau O, Pillet L, Inaebnit A, Borens , Quinn TM. Biomechanical characterization and in vitro mechanical injury of eld- 
erly human femoral head cartilage: comparison to adult bovine humeral head cartilage. OsteoArthritis and Cartilage $2006 ; 14: 589-596$.

[9] Patwari P, Cook MN, DiMicco MA, Blake SM, James IE, Kumar S, Cole AA, Lark MW, Grodzinsky AJ. Proteoglycan degradation after injurious compression of bovine and human articular cartilage in vitro: interaction with exogenous cytokines. Arthritis Rheum 2003;48:1292-301.

[10] Hodge WA, Fujan RS, Carlson KL, Burgess RG, Harris WH, Mann RW. Contact pressures in the human hip joint measured in vivo. PNAS 1986;83:2879-2883.

[11] Stoltz JF, de Isla N, Huselstein C, Bensoussan D, Muller S, Decot V. Mechanobiology and cartilage engineering: the underlying pathophysiological phenomena. Biorheology 2006; 43:171-80.

[12] Hung CT, Mauck RL., Wang CC, Lima EG, Ateshian GA. A Paradigm for Functional Tissue Engineering of Articular Cartilage via Applied Physiologic Deformational Loading. Annals of Biomedical Engineering 2004;32:35-49.

[13] Lee MS, Trindade MC, Ikenoue T, Schurman DJ, Goodman SB, Smith RL. Effects of shear stress on nitric oxide and matrix protein gene expression in human osteoarthritic chondrocytes in vitro. J Orthop Res 2002;20:556-61.
[14] Fermor B, Weinberg JB, Pisetsky DS, Misukonis MA, Banes AJ, Guilak F. The effects of static and intermittent compression on nitric oxide production in articular cartilage explants. J Orthop Res 2001;19:729-37.

[15] Gosset M, Berenbaum F, Levy A, Pigenet A, Thirion S, Saffar $\mathrm{JL}$, Jacques C Prostaglandin E2 synthesis in cartilage explants under compression: mPGES-1 is a mechanosensitive gene. $\mathrm{Ar}-$ thritis Res Ther. 2006;8:R135.

[16] Agarwal S, Long P, Gassner R, Piesco NP, Buckley MJ. Cyclic Tensile Strain Suppresses Catabolic Effects of Interleukin-1b in Fibrochondrocytes From the Temporomandibular Joint Arthritis \& Rheumatism 2001;44:608-617.

[17] Lee DA, Frean SP, Lees P, Bader DL. Dynamic mechanical compression influences nitric oxide production by articular chondrocytes seeded in agarose. Biochem Biophys Res Commun. 1998;251:580-585.

[18] Piscoya JL, Fermor B, Kraus VB, Stabler TV, Guilak F. The influence of mechanical compression on the induction of osteoarthritis-related biomarkers in articular cartilage explants. OsteoArthritis and Cartilage 2005;13:1092-1099.

[19] Kurz B, Lemke AK, Fay J, Pufe T, Grodzinsky AJ, Schunke M. Pathomechanisms of cartilage destruction by mechanical injury. Annals of Anatomy 2005;187:473-485. 